\title{
EXAME CITOPATOLÓGICO DO COLO DO ÚTERO: FAIXA ETÁRIA E RESULTADOS ENCONTRADOS
}

\section{CERVICAL CYTOPATHOLOGICAL EXAMINATION: AGE RANGE AND RESULTS FOUND}

\section{EXAMEN CITOPATOLÓGICO DEL CERVIX: RANGO DE EDAD Y RESULTADOS ENCONTRADOS}

\begin{abstract}
Anne Gabriella Pacito Monteiro ${ }^{1}$, Heloyse Nathelly Rodrigues Dutra ${ }^{2}$, Tatiane Silva Castellini ${ }^{3}$, Juliana da Silva
\end{abstract} Vigo $^{4}$, Daiane Cortêz Raimondi ${ }^{5}$

Como citar esse artigo: Monteiro AGP, Dutra HNR, Castellini TS, Vigo JS, Raimondi DC. Exame citopatológico do colo do útero: faixa etária e resultados encontrados. Rev Enferm Atenção Saúde [Internet]. 2021 [acesso em:__]; 10(3):e202133. doi: https://doi.org/ 10.18554/reas.v10i3.4562

\section{RESUMO}

Objetivo: Identificar a idade e os resultados dos exames citopatológicos do colo do útero de mulheres atendidas em uma unidade de saúde. Metodologia: Trata-se de uma pesquisa retrospectiva documental realizada através dos livros de registro do exame de 2016 a 2018, onde foram coletados a idade e os resultados dos exames. Resultados: Pode-se constatar que a maioria dos exames obtiveram resultados normais, seguidos de inflamação sem agente etiológico e Gardnerella vaginalis. Apenas $0,7 \%$ das mulheres apresentaram lesões intraepiteliais, de baixo e alto grau, sendo mais frequente entre mulheres com idade inferior a 25 anos. Em relação à idade, a maior adesão foi entre as mulheres de 50 a 59 anos. Conclusões: Dentre a faixa etária recomendada para o exame, pode-se constatar que a maioria das mulheres obtiveram resultados normais, reforçando assim a importância do exame na prevenção e detecção precoce do câncer do colo do útero.

Descritores: Teste de Papanicolaou. Neoplasias do Colo do Útero. Avaliação de Resultados em Cuidados de Saúde. Enfermagem.

\footnotetext{
1 UNIPAR - Universidade Paranaense. Discente do $5^{\circ}$ ano de Enfermagem pela UNIPAR - Universidade Paranaense. Participante do Programa de Iniciação Científica (PIC).

${ }^{2}$ Universidade Paranaense. Discente do $5^{\circ}$ ano de Enfermagem pela UNIPAR - Universidade Paranaense.

3 UNIPAR - Universidade Paranaense. Discente do $5^{\circ}$ ano de Enfermagem pela UNIPAR - Universidade Paranaense.

${ }^{4}$ UNIPAR - Universidade Paranaense. Discente do $5^{\circ}$ ano de Enfermagem pela UNIPAR - Universidade Paranaense.

${ }^{5}$ UNIPAR - Universidade Paranaense. Mestre em Enfermagem pela Universidade Estadual de Maringá (UEM). Especialista em Auditoria em Saúde pela Faculdade Ingá. Especialista em Docência do Ensino Superior pela Unicesumar. Graduada em Enfermagem pela UNIPAR, laureada melhor aluna do Curso de Enfermagem da Universidade Paranaense. Foi professora assistente (Tempo Integral - 2013 - 2015) dos Cursos de Enfermagem, Farmácia e Fisioterapia; Coordenadora do Curso de graduação em Enfermagem da Universidade Paranaense UNIPAR - Unidade Universitária Toledo -PR (2013-01/2015). Docente dos Cursos de Enfermagem e Medicina da Unipar - Umuarama (2019). Atualmente é Docente e Coordenadora do Curso de Enfermagem da UNIPAR e Gestora Pedagógica dos Cursos de Especialização na Área da Saúde e Desportivo na modalidade de Ensino à Distância - EAD da UNIPAR - Unidade Universitária de Umuarama - PR. Atua na área de atenção primária à saúde e saúde da mulher. Enfermeira da Estratégia Saúde da Família no Município de Umuarama - PR
} 


\begin{abstract}
Objective: To identify the age and results of cervical cytopathological examinations of women treated at a health unit. Methodology: This is a retrospective documentary research carried out through the exam registration books from 2016 to 2018, where age and test results were collected. Results: It can be seen that most of the tests obtained normal results, followed by inflammation without etiologic agent and Gardnerella vaginalis. Only $0.7 \%$ of women had low- and high-grade intraepithelial lesions, being more frequent among women under the age of 25 years. Regarding age, the highest adherence was among women aged 50 to 59 years. Conclusions: Among the age range recommended for the exam, it can be seen that most women obtained normal results, thus reinforcing the importance of the exam in the prevention and early detection of cervical cancer.
\end{abstract}

Descriptors: Papanicolaou Test. Uterine Cervical Neoplasms. Outcome Assessment, Health Care. Nursing.

\title{
RESUMEN
}

Objetivo: Identificar la edad y los resultados de los exámenes citopatológicos cervicales de mujeres tratadas en una unidad de salud. Metodología: Esta es una investigación documental retrospectiva realizada a través de los libros de registro de exámenes de 2016 a 2018, donde se recopilaron los resultados de la edad y las pruebas. Resultados: se puede observar que la mayoría de los exámenes obtuvieron resultados normales, seguidos de inflamación sin agente etiológico y Gardnerella vaginalis. Solo el $0.7 \%$ de las mujeres tenían lesiones intraepiteliales de bajo y alto grado, siendo más frecuente entre las mujeres menores de 25 años. En cuanto a la edad, la adherencia más alta fue entre mujeres de 50 a 59 años. Conclusiones: Entre el rango de edad recomendado para el examen, se puede ver que la mayoría de las mujeres obtuvieron resultados normales, lo que refuerza la importancia del examen en la prevención y detección temprana del cáncer cervical.

Descriptoress: Prueba de Papanicolaou. Neoplasias del Cuello Uterino. Evaluácion de Resultado en la Atención de Salud. Enfermería.

\section{INTRODUÇÃO}

O câncer do colo do útero constitui um grave problema de saúde pública em países em desenvolvimento. No Brasil é o terceiro tumor mais incidente entre as mulheres, estando atrás apenas do câncer de mama e colorretal, constituindo a quarta causa de óbito na população feminina. ${ }^{1}$

De acordo com o Instituto Nacional de Câncer José Alencar Gomes da Silva $\mathrm{INCA}^{1}$ há estimativa de 16.590 novos casos de câncer do colo do útero no Brasil para 2020, ressalta-se assim a gravidade da doença, visto que em 2017 foi responsável por 10.326 óbitos de mulheres brasileiras, no qual 6.385 estavam diretamente relacionados ao colo uterino.

O câncer do colo do útero pode ocasionar sangramento vaginal, secreção anormal e dores abdominais, relacionado a condições intestinais e urinárias em casos mais avançados. ${ }^{2}$ É válido mencionar que a história do câncer do colo do útero, na maioria das vezes apresenta um extenso período de lesões precursoras, 
assintomáticas, sendo curáveis na quase totalidade dos casos quando tratadas adequadamente, $\mathrm{o}$ que reforça a importância do diagnóstico precoce com a realização do exame preventivo do câncer do colo uterino. ${ }^{3}$

Houve a descoberta que para o desenvolvimento do câncer de colo do útero é necessário que a mulher apresente infecção pelo papilomavírus humano (HPV), sendo os tipos HPV16 e o HPV18 mais comuns para esta patologia. Além desta infecção, há como fatores de risco para o câncer de colo uterino: múltiplos parceiros, genética, imunidade, idade, tabagismo entre outros. ${ }^{2}$

Em relação à prevenção do câncer de colo do útero, destaca-se a vacinação contra o papilomavírus humano que é disponibilizada gratuitamente nas unidades de saúde, além da importância de práticas de educação em saúde na atenção primária para comunidade, a fim de sensibilizar as mulheres a hábitos de vida adequados que promovam a saúde das mesmas. ${ }^{4-5}$ Sobre a redução da incidência e a mortalidade da doença, a principal estratégia é a realização periódica do exame citopatológico do colo do útero nas mulheres de 25 a 64 anos, que visa diagnosticar precocemente o câncer de colo uterino e lesões precursoras, deste modo orientar e incentivar as mulheres para realização do exame é fundamental para prevenção da doença. ${ }^{3-6}$
Mesmo diante da relevância do exame citopatológico do colo uterino na prevenção e detecção precoce da doença, observa-se que as mulheres possuem dificuldades de adesão ao exame, sendo que os motivos para não realização estão relacionados com o desconhecimento da importância do procedimento, baixa escolaridade, crenças, medo do exame e do possível diagnóstico da doença, a vergonha e constrangimento, o não reconhecimento de ser integrante do grupo de risco, o nível socioeconômico e cultural dentre outros. ${ }^{7-8}$ Isto reflete a importância de disseminar informações referentes a realização do exame e sua relevância na prevenção do câncer do colo do útero.

É válido mencionar que é responsabilidade da equipe da atenção primária realizar ações preventivas do câncer do colo do útero através de atividades de educação em saúde, busca ativa, imunização, além da detecção precoce do câncer e de lesões por meio da realização do exame citopatológico do colo do útero, sendo este realizado pelo médico ou enfermeiro nas unidades de saúde. ${ }^{3}$

Dentre as ações desenvolvidas pelo enfermeiro, salienta-se a assistência integral às mulheres do território de abrangência da unidade de saúde sob sua responsabilidade, desde orientações sobre prevenção primária e secundária do câncer do colo do útero, ou seja, da sensibilização 
para promoção de hábitos de vida saudáveis até a realização da consulta de enfermagem, coleta de exame citopatológico do colo uterino e o encaminhamento em caso de alterações citológicas. $^{7,5}$

Devido a elevada incidência e mortalidade decorrente do câncer de colo uterino, verifica-se a importância de identificar o perfil das mulheres que realizam o exame preventivo do câncer de colo uterino, além de identificar os resultados encontrados, tendo por base que este exame é primordial para prevenção secundária, no rastreamento de neoplasias, na identificação e tratamento precoce de lesões, proporcionando assim a análise da necessidade de atividades de sensibilização da população para adesão do exame.

Diante do exposto este estudo objetiva identificar a idade das mulheres que realizaram exame citopatológico do colo do útero entre os anos de 2016 a 2018, em uma Unidade de Saúde Escola da Região Sul do Brasil, bem como identificar os resultados encontrados.

\section{METODOLOGIA}

Trata-se de uma pesquisa retrospectiva documental realizada através da análise de livros de registro do exame citopatológico do colo do útero de uma Unidade Básica de Saúde Escola da região
Sul do Brasil. Salienta-se que a pesquisa documental refere-se ao acesso a documentos originais em busca de informações para serem analisadas cientificamente. Este método elimina a intervenção do pesquisador nos dados da pesquisa. $^{9}$

A coleta de dados foi realizada entre abril e junho de 2019, no qual a amostra foi composta pelas mulheres que realizaram o exame nos últimos três anos (2016 a 2018) na referida unidade de saúde.

Para o desenvolvimento da pesquisa, primeiramente foi solicitado autorização da Secretaria Municipal de Saúde. Após a autorização e de posse deste documento, o projeto de pesquisa foi cadastrado na Plataforma Brasil para apreciação e parecer pelo Comitê Permanente de Ética em Pesquisas Envolvendo Seres Humanos (COPEP) da Universidade Paranaense UNIPAR, sendo aprovado pelo parecer CAAE 94762318.4.0000.0109. Foi solicitado e aprovado a dispensa do Termo de Consentimento Livre Esclarecido TCLE, visto que se trata de um estudo não intervencionista com utilização de dados de livros de registros que não permite a identificação das usuárias participantes.

Logo após a aprovação do projeto de pesquisa pelo comitê de ética, a coordenadora das unidades básicas do município e a enfermeira responsável pela 
unidade foram comunicadas sobre o início da pesquisa. Assim, os acadêmicos do curso de enfermagem participantes do Projeto de Iniciação Científica - PIC de uma Universidade Privada do Noroeste do Paraná juntamente com a professora orientadora iniciaram a coleta de dados, onde as informações (idade e resultado) obtidas foram transcritas para planilha do softwareMicrosoft Office Excel, no qual posteriormente foi realizada a análise por meio da estatística descritiva.

Cabe mencionar que o projeto de pesquisa cumpriu todas as exigências éticas estabelecidas na Resolução CNS $\mathrm{n}^{0}$. $466 / 2012$.

\section{RESULTADOS}

Diante da coleta de dados, pode-se constatar que foram realizados 1.278 exames citopatológicos do colo uterino, no qual 502 exames foram realizados em 2016, 384 exames em 2017 e 392 em 2018.

Destaca-se na Tabela $01 \quad 0$ quantitativo de mulheres que realizaram o exame preventivo segundo faixa etária.

Tabela 1 - Descrição das mulheres que realizaram o exame preventivo do colo uterino segundo idade e ano de realização do exame.

\begin{tabular}{|c|c|c|c|c|c|c|}
\hline \multirow{2}{*}{ Idade } & \multicolumn{2}{|c|}{ Mulheres2016 } & \multicolumn{2}{|c|}{ Mulheres2017 } & \multicolumn{2}{|c|}{ Mulheres2018 } \\
\hline & $\mathbf{n}$ & $\%$ & $\mathbf{n}$ & $\%$ & $\mathbf{n}$ & $\%$ \\
\hline Abaixo de 25 anos & 58 & 11,5 & 63 & 16,4 & 50 & 12,8 \\
\hline 25 a 29 anos & 43 & 8,6 & 23 & 6,0 & 35 & 8,9 \\
\hline 30 a 39 anos & 82 & 16,3 & 74 & 19,3 & 53 & 13,5 \\
\hline 40 a 49 anos & 95 & 18,9 & 76 & 19,8 & 79 & 20,2 \\
\hline 50 a 59 anos & 127 & 25,3 & 92 & 24,0 & 95 & 24,2 \\
\hline 60 a 64 anos & 42 & 8,4 & 27 & 7,0 & 39 & 9,9 \\
\hline 65 a 69 anos & 26 & 5,2 & 12 & 3,1 & 17 & 4,3 \\
\hline 70 a 79 anos & 21 & 4,2 & 7 & 1,8 & 21 & 5,4 \\
\hline 80 anos ou mais & 1 & 0,2 & 3 & 0,8 & 3 & 0,8 \\
\hline Não constava idade & 7 & 1,4 & 7 & 1,8 & 0 & 0 \\
\hline Total & 502 & 100 & 384 & 100 & 392 & 100 \\
\hline
\end{tabular}


Verifica-se na Tabela 1 que houve maior procura das mulheres entre 50 a 59 anos para a realização do exame citopatológico do colo do útero em todos os anos, sendo seguidas pelas mulheres entre a faixa etária de 40 a 49 anos e posteriormente de 30 a 39 anos. Cabe mencionar que sete exames não constavam idade da mulher.

A fim de identificar os resultados encontrados nos exames citopatológicos do colo uterino realizados na unidade de saúde estudada entre 2016 a 2018 foi realizado a Tabela 2 .

Tabela 2 - Descrição dos resultados do exame citopatológico do colo do útero de 2016 a 2018.

\section{Resultados dos exames citopatológicos do colo do útero}

\begin{tabular}{lcc} 
Conclusão do exame & N & \% \\
\hline Dentro dos limites de normalidade & 635 & 49,7 \\
Inflamação sem agente etiológico & 295 & 23,1 \\
Bacilos supracitoplasmáticos sugestivos de Gardnerella vaginalis & 163 & 12,8 \\
Bacilos supracitoplasmáticos sugestivos de Candida albicans & 65 & 5,1 \\
Atrofia com inflamação & 33 & 2,5 \\
Lesão intraepitelial escamosa de baixo grau (LSIL) & 06 & 0,5 \\
Lesão intraepitelial escamosa de alto grau (HSIL) & 03 & 0,2 \\
Amostra insuficiente & 02 & 0,2 \\
Sem anotação resultados & 76 & 5,9 \\
Total & 1.278 & 100 \\
\hline
\end{tabular}

Fonte: Autoras, 2019.

Diante da Tabela 2, pode-se observar que $635(49,7 \%)$ mulheres obtiveram resultados normais, $295 \quad(23,1 \%)$ apresentaram inflamação sem agente etiológico e $163(12,8 \%)$ exames foram sugestivos de Gardnerella vaginalis. Destaca-se que apenas nove $(0,7 \%)$ mulheres apresentaram lesões intraepiteliais, sendo elas de baixo $(0,5 \%)$ e alto grau $(0,2 \%)$.

Constata-se ainda na Tabela 02 que $76(5,9 \%)$ exames não constavam anotação do resultado no livro de registros da unidade de saúde estudada.

Com intuito de identificar os resultados de exames preventivos 
conforme faixa etária recomendada para realização do procedimento, ou seja, nas mulheres entre 25 a 64 anos, foi realizada a classificação etária conforme consta na Tabela 3.

Tabela 3 - Descrição dos resultados dos exames citopatológicos do colo uterino segundo faixa etária.

Idade das mulheres que realizaram exame preventivo

\begin{tabular}{lccccccccccc}
$\begin{array}{c}\text { Resultado do Exame } \\
\text { citopatológico do colo do } \\
\text { útero }\end{array}$ & $\begin{array}{c}\text { Abaixo de } \\
\mathbf{2 5} \text { anos }\end{array}$ & $\begin{array}{c}\mathbf{2 5} \text { a } \mathbf{6 4} \\
\text { anos }\end{array}$ & $\begin{array}{c}\mathbf{6 5} \text { a 79 } \\
\text { anos }\end{array}$ & $\begin{array}{c}\mathbf{8 0} \text { anos } \\
\text { ou mais }\end{array}$ & $\begin{array}{c}\text { Não } \\
\text { consta } \\
\text { idade }\end{array}$ \\
& $\mathbf{N}$ & $\mathbf{\%}$ & $\mathbf{N}$ & $\mathbf{\%}$ & $\mathbf{N}$ & $\mathbf{\%}$ & $\mathbf{N}$ & $\mathbf{\%}$ & $\mathbf{N}$ & $\%$ \\
\hline $\begin{array}{l}\text { Dentro dos limites de } \\
\text { normalidade }\end{array}$ & 64 & 37,4 & 508 & 51,7 & 50 & 48,1 & 05 & 71,4 & 08 & 57,1 \\
Inflamação sem agente & 44 & 25,7 & 225 & 23,0 & 20 & 19,2 & 01 & 14,3 & 05 & 35,8 \\
etiológico & & & & & & & & & & \\
Gardnerella vaginalis & 30 & 17,5 & 124 & 12,6 & 07 & 6,7 & 01 & 14,3 & 01 & 7,1 \\
Candida albicans & 19 & 11,1 & 44 & 4,5 & 02 & 1,9 & 00 & 0 & 00 & 0 \\
Atrofia com inflamação & 00 & 0 & 18 & 1,8 & 15 & 14,4 & 00 & 0 & 00 & 0 \\
LSIL & 05 & 3,0 & 01 & 0,1 & 00 & 0 & 00 & 0 & 00 & 0 \\
HSIL & 01 & 0,6 & 02 & 0,2 & 00 & 0 & 00 & 0 & 00 & 0 \\
Amostra insuficiente & 00 & 0 & 01 & 0,1 & 01 & 1,0 & 00 & 0 & 00 & 0 \\
Sem anotação resultados & 08 & 4,7 & 59 & 6,0 & 09 & 8,7 & 00 & 0 & 00 & 0 \\
Total & 171 & 100 & 982 & 100 & 104 & 100 & 07 & 100 & 14 & 100 \\
\hline
\end{tabular}

Fonte: Autoras, 2019.

Diante da Tabela 03, pode-se constatar que na faixa etária preconizada para realização do exame, ou seja, de 25 a 64 anos, a maioria das mulheres $(51,7 \%)$ apresentaram resultados de exames normais, seguidos por alterações benignas como inflamação sem agente etiológico e os achados microbiológicos de bacilos sugestivos de Gardnerella Vaginalis, sendo que $02 \quad(0,2 \%)$ mulheres apresentaram lesão intraepitelial escamosa de alto grau (HSIL) e apenas $01(0,1 \%)$ mulher apresentou lesão intraepitelial escamosa de baixo grau (LSIL). As mulheres com idade inferior a 25 anos tiveram uma maior propensão a lesões 
(3,6\%) bem como alterações benignas, diferentemente das mulheres acima de 65 anos que apresentaram apenas resultados normais e alterações benignas.

\section{DISCUSSÃO}

As mulheres de 25 a 64 anos apresentaram uma maior adesão ao exame citopatológico do colo uterino, provavelmente devido ser a faixa etária preconizada pelo Ministério da Saúde para realizar o procedimento, além de ser a faixa etária mais extensa quando comparada as demais idades. É notório a maior procura para realização do exame entre as mulheres de 50 a 59 anos, seguidas pelas mulheres de 40 a 49 anos, e isto pode estar relacionado com o fato de ser a faixa etária mais acometida pela doença.

Esses dados divergem da pesquisa realizada em Sergipe cuja faixa etária com maior adesão ao exame citopatológico do colo do útero foi entre as mulheres de 29 a 34 anos. $^{10}$

Há contraste também com estudo brasileiro realizado no Estado do Rio Grande do Sul onde identificaram aumento da realização do exame proporcional a idade da mulher, sendo mais frequente acima de 55 anos. ${ }^{11}$

Acredita-se que a sensibilização das mulheres da faixa etária de 25 a 64 anos para realização do exame pelas equipes de saúde do município estudado tem contribuído para adesão desta população ao exame.

Sobre os resultados dos exames, nota-se que de modo geral $49,7 \%$ das mulheres apresentaram resultados dentro dos limites de normalidade e quando avaliado apenas as mulheres dentro da faixa etária recomendada para realização do exame citopatológico do colo uterino a proporção de exames normais alcançaram $51,7 \%$. Estes resultados foram seguidos por inflamação sem agente etiológico como alteração celular benigna mais prevalente e achados microbiológicos: bacilos supracitoplasmáticos sugestivos de Gardnerella vaginalis.

Ainda sobre os resultados, pode-se afirmar que poucas mulheres apresentaram lesões possivelmente precursoras do câncer de colo uterino, sendo que a maior porcentagem foi entre as mulheres abaixo da faixa etária recomendada para o exame, ao contrário das mulheres acima de 65 anos que não obtiveram nenhuma lesão.

As lesões em mulheres abaixo de 25 anos pode estar relacionada com os hábitos de vida, a iniciação precoce das práticas sexuais, múltiplos parceiros dentre outros, ${ }^{3}$ sendo fundamental a prática da educação em saúde voltada a esta população, visando sensibilizar as mulheres para mudanças que objetivem reduzir a exposição das mesmas a doenças. 
Salienta-se que as mulheres com menos de 25 anos constituem grupo de risco para infecção do HPV, no entanto, geralmente apresentam resolução espontânea, o que justifica a realização do acompanhamento das que apresentam alterações no exame preventivo sem intervenções até os 25 anos. ${ }^{3}$

Os dados encontrados divergem da pesquisa de Ströher e colaboradores ${ }^{12}$ desenvolvida com mulheres que realizaram o exame preventivo em uma unidade de Saúde de Uruguaiana no Rio Grande do Sul, no qual entre os resultados de exames alterados identificaram maior prevalência de atipias de células escamosas de significado indeterminado (ASC-US), seguida das lesões intraepiteliais escamosa de baixo grau (LSIL). No entanto, assemelha-se ao presente estudo ao demonstrar que as alterações foram mais frequentes nas mulheres com idade abaixo de 25 anos.

Uma pesquisa realizada na região Sul do Brasil identificou que $34,7 \%$ dos exames preventivos realizados tiveram resultado dentro dos limites de normalidade, apresentando-se abaixo do presente estudo, porém corrobora ao identificar as alterações inflamatórias como as mais frequentes nos resultados do exame citopatológico do colo uterino e uma baixa porcentagem de lesões intraepiteliais de baixo e alto grau. ${ }^{11}$
Corroborando com os dados apresentados, o estudo de Leitão ${ }^{13}$ menciona que o agente patogênico mais prevalente nos resultados de exames preventivos estudados foram a Gardnerella vaginalis $(28,3 \%)$, seguido pelos cocos e bacilos (24,8\%), Candida sp. $(7,7 \%)$ e Trichomonas vaginalis( $0,5 \%)$.

Ainda sobre a microbiologia, uma pesquisa realizada no Piauí com mulheres que apresentaram alterações citopatológicas, constatou os cocos como o agente microbiológico mais prevalente nos resultados dos exames, diferentemente do presente estudo onde prevaleceu os bacilos supracitoplasmáticos sugestivos de Gardnerella vaginalis. ${ }^{14}$

Cabe mencionar que no decorrer dos três anos estudados nenhuma mulher apresentou carcinoma, isto pode estar relacionado com hábitos de vida saudáveis das mulheres e realização periódica do exame citopatológico do colo uterino, visto que pode detectar lesões iniciais que tratadas previnem o câncer do colo do útero. Porém, como o presente estudo não avaliou a periodicidade e cobertura de realização do exame, esses dados também podem relacionar a falha no acesso e na cobertura do exame citopatológico do colo uterino, pois mulheres que possuem alterações podem não terem realizados o exame neste período. 
É válido salientar que não foi possível avaliar a cobertura do exame na referida unidade, visto que conforme relatado pela enfermeira responsável da UBS houve alteração do sistema eletrônico da Unidade, o que impossibilitou o relatório da faixa etária da área de abrangência nos anos estudados.

É indispensável enfatizar que no livro de registro constava a ausência de dados como data de nascimento e resultados dos exames. Isto deve ser revisto e evitado, sendo necessário a organização, pois a inserção desses dados é de grande importância para o planejamento do cuidado integral à saúde.

\section{CONCLUSÃO}

Constata-se com os resultados do presente estudo que as mulheres de 50 a 59 anos foram prevalentes na realização do exame, no qual a maioria dos resultados encontra-se dentro dos limites de normalidade e com alterações benignas, sendo que as lesões precursoras foram mais incidentes nas mulheres com idade inferior a 25 anos.

Cabe reforçar a necessidade de conscientizar as mulheres sobre a importância da realização do exame preventivo periodicamente para prevenção e detecção precoce do câncer de colo do útero, bem como incentivá-las a adesão de hábitos de vida saudáveis e prática sexual segura.

Vale ainda salientar sobre as ausências de informações nos livros de registro, sendo fundamental rever e organizar os procedimentos referentes ao exame, a fim de proporcionar o planejamento de ações e consequentemente a qualidade da assistência prestada.

Em relação às limitações, pode-se destacar o desenvolvimento do estudo em apenas uma unidade de saúde, bem como a não avaliação da periodicidade e cobertura do exame citopatológico do colo uterino.

\section{REFERÊNCIAS}

1. Instituto Nacional de Câncer José Alencar Gomes da Silva. Tipos de câncer: câncer do colo do útero [Internet]. 2021 [acesso em 05 fev 2020]. Disponível em: https://www.inca.gov.br/tipos-decancer/cancer-do-colo-doutero\#: :text=O\%20c\%C3\%A2ncer\%20do $\% 20$ colo $\% 20$ do,das $\% 20$ vezes $\% 20$ n $\%$ C3\% A3o\%20causa\%20doen\%C3\%A7a 2. Ministério da Saúde (Brasil), Secretaria de Atenção à Saúde, Departamento de Atenção Básica. Controle dos cânceres do colo do útero e da mama [Internet]. 2ed. Brasília, DF: Ministério da Saúde; 2013 [citado em $17 \mathrm{dez}$ 2021]. 124 p. (Cadernos de Atenção Básica; n. 13). Disponível em: https://bvsms.saude.gov.br/bvs/publicacoes /controle_canceres_colo_utero_2013.pdf

3. Instituto Nacional de Câncer José Alencar Gomes da Silva, Coordenação de Prevenção e Vigilância, Divisão de Detecção Precoce e Apoio à Organização de Rede. Diretrizes brasileiras para o rastreamento do câncer do colo do útero 
[Internet]. 2ed rev atual. Rio de Janeiro: INCA; 2016 [citado em $17 \mathrm{dez}$ 2021]. 114 p. Disponível em:

https://www.inca.gov.br/sites/ufu.sti.inca.l ocal/files//media/document//diretrizesparao rastreamentodocancerdocolodoutero_2016 _corrigido.pdf

4. Ministério da Saúde (Brasil), Secretaria de Vigilância em Saúde, Departamento de Vigilância de Doenças Transmissíveis, Coordenação-Geral do Programa Nacional de Imunizações. Informe técnico da ampliação da oferta das vacinas papilomavírus humano $6,11,16$ e 18 (recombinante) - vacina HPV quadrivalente e meningocócica $\mathrm{C}$ (conjugada). Brasília, DF: Ministério da Saúde; 2018.

5. Melo MCSC, Vilela F, Salimena AMO, Souza IEO. O enfermeiro na prevenção do câncer do colo do útero: o cotidiano da atenção primária. Rev Bras Cancerol. [Internet]. 2012 [citado em $17 \mathrm{dez}$ 2021]; 58(3):389-98. Disponível em:

https://rbc.inca.gov.br/site/arquivos/n_58/v 03/pdf/08_artigo_enfermeiro_prevencao_c ancer_colo_utero_cotidiano_atencao_prim aria.pdf

6. Ministério da Saúde (Brasil), Instituto

Sírio-Libanês de Ensino e Pesquisa

Protocolos da atenção básica: saúde das mulheres [Internet]. Brasília, DF:

Ministério da Saúde; 2016 [citado em 17 dez 2021]. Disponível em:

https://bvsms.saude.gov.br/bvs/publicacoes /protocolos_atencao_basica_saude_mulher es.pdf

7. Carvalho LRS, Jurado SR. Motivos que influenciam a não realização do exame de Papanicolaou. Rev Recien, [Internet]. 2018 [citado em $17 \mathrm{dez} 2021$ ]; 8(23):39-46.

Disponível em:

https://www.recien.com.br/index.php/Reci en/article/view/259/pdf_1

8. Amorim LTL, Monteiro NJ, Nogueira LMV, Rodrigues ILA, André SR. Exame de colpocitologia oncótica: revisão integrativa. Rev Enferm Atenção Saúde [Internet]. jan/jul 2018 [citado em $17 \mathrm{dez}$ 2021]; 7(1):209-24. Disponível em: http://seer.uftm.edu.br/revistaeletronica/ind ex.php/enfer/article/view/2436/pdf

9. Sá-Silva JR, Almeida CD, Guindani JF.

Pesquisa documental: pistas teóricas e metodológicas. Rev Bras Hist Ciênc Soc. [Internet]. jul 2009 [citado em $17 \mathrm{dez}$ 2021]; 1(1):2-15. Disponível em: https://periodicos.furg.br/rbhcs/article/view $/ 10351 /$ pdf

10. Reis NROG, Costa AMC, Madi RR, Melo CM. Perfil microbiológico e alterações citológicas associadas ao material cérvico-vaginal coletado em consultório de enfermagem, de 2009 a 2011 em Aracaju / SE. Scientia Plena [Internet]. maio 2013 [citado em $17 \mathrm{dez}$ 2021]; 9(5):2-8. Disponível em:

https://www.scientiaplena.org.br/sp/article/ view/1142/772

11. Fredrich EK, Renner JDP. Cervical cytopathological changes in Pap smear test in the city of Santa Cruz do Sul, Rio Grande do Sul, Brazil. J Bras Patol Med Lab. [Internet]. 2019 [citado em $17 \mathrm{dez}$ 2021]; 55(3):246-57. Disponível em: https://www.scielo.br/j/jbpml/a/QkqRQ37d gGZDGzcTLBsZrwk/?format=pdf\&lang=e $\mathrm{n}$

12. Strõher DJ, Aramburu TDB, Abad MAS, Nunes VT, ManfrediniV. Perfil citopatológico de mulheres atendidas nas unidades básicas do município de Uruguaiana, RS. DST J Bras Doenças Sex Transm. [Internet]. 2012 [citado em $17 \mathrm{dez}$ 2021]; 24(3):167-70. Disponível em: http://www.dst.uff.br/revista24-3-2012/3Perfil_Citopatologico_de_Mulheres.pdf 13. Leitão NMA, Pinheiro AKB, Anjos SJSB, Vasconcelos CTM, Nobre RNS. Avaliação dos laudos citopatológicos de mulheres atendidas em um serviço de enfermagem ginecológica. REME Rev. Min. Enferm. [Internet]. out/dez 2008 [citado em 05 nov 2019]; 12(4):508-15. Disponível em:

https://cdn.publisher.gn1.link/reme.org.br/ pdf/v12n4a09.pdf

14. Sousa ACO, Passos FFV, Costa GS, Oliveira FP, Rodrigues TS. Análise das alterações citopatológicas registradas no 
sistema de informação do câncer de colo do útero em Teresina. Revista

Interdisciplinar [Internet]. 2017 [citado em

17 dez 2021]; 10(4):21-30. Disponível em:

RECEBIDO: $25 / 05 / 2020$

APROVADO: 14/01/2021

PUBLICADO: dez/2021 https://dialnet.unirioja.es/servlet/articulo?c odigo $=6772037$ 